\title{
Rotation of an erupting filament observed by STEREO EUVI and COR1 instruments (Corrigendum) ${ }^{\star}$
}

\author{
A. Bemporad ${ }^{1}$, M. Mierla ${ }^{2}$, and D. Tripathi ${ }^{3}$ \\ ${ }^{1}$ INAF - Turin Astronomical Observatory, Pino Torinese (TO), Italy \\ e-mail: bemporad@oato.inaf.it \\ 2 Royal Observatory of Belgium, Brussels, Belgium Institute of Geodynamics of the Romanian Academy, Bucharest, Romania \\ Research Center for Atomic Physics and Astrophysics, Faculty of Physics, University of Bucharest, Romania \\ e-mail: marilena@oma.be \\ 3 Department of Applied Mathematics and Theoretical Physics, University of Cambridge, Wilberforce Road, Cambridge CB3 0WA, \\ UK \\ e-mail: durgesh@iucaa.ernet.in
}

A\&A 531, A147 (2011), DOI: 10.1051/0004-6361/201016297

Key words. Sun: filaments, prominences - Sun: corona - Sun: coronal mass ejections (CMEs) - errata, addenda

Results on the erupting prominence sense of rotation and on the handedness of the smal-scale helix are confirmed. Nevertheless, our conclusion that "The sense of rotation of this helix agrees with the observed prominence rotation, providing evidence for the conversion of twist into writhe" is not correct.

In the paper we mentioned that the (left) handedness of the small-scale helix agrees with the observed large-scale (clock-wise) sense of rotation of the erupting prominence, supporting the conversion of twist into writhe of the same sign in a kink-unstable magnetic flux-rope. This sentence is not correct, because a left-handed (right-handed) flux rope transforming its twist into writhe helicity will build up a left-handed (righthanded) writhe helicity (by helicity conservation), which it will show up as a counter clock-wise (clock-wise) rotation of the rising flux rope (see e.g. Török \& Kliem 2005; Török et al. 2010). Hence, results described in the paper do not support the idea that the observed rotation occurs as a consequence of conversion of twist into writhe, by assuming that $H$ is a conserved quantity. The large-scale handedness of the whole prominence (hence, not only in the small-scale helix) is now being carefully investigated with $\mathrm{H} \alpha$ images, magnetic field extrapolations, and 3D reconstructions of prominence threads. Alternative mechanisms leading to the observed clock-wise rotation and explanations for the formation of the observed small-scale left-handed helix will be provided and discussed in a forthcoming paper.

Acknowledgements. The authors acknowledge Bernhard Kliem and Tibor Török for pointing out this inconsistency and for very useful discussions on the kink instability model.

\section{References}

Török, T., \& Kliem, B. 2005, ApJ, 630, L97

Török, T., Berger, M. A., \& Kliem, B. 2010, A\&A, 516, A49

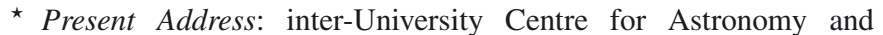
Astrophysics, Post Bag 4, Ganeshkhind, 411007 Pune, India. 